Article

\title{
Polyamide 6/Multiwalled Carbon Nanotubes Nanocomposites with Modified Morphology and Thermal Properties
}

\author{
Nasir Mahmood ${ }^{1}$, Mohammad Islam ${ }^{2,3, *}$, Asad Hameed ${ }^{4}$ and Shaukat Saeed ${ }^{5}$ \\ 1 Department of Materials Science and Engineering, College of Engineering, Peking University, \\ Beijing 100871, China; E-Mail: nasirm38@gmail.com \\ 2 College of Engineering, King Saud University, P.O. Box 800, Riyadh 11421, Saudi Arabia \\ 3 School of Chemical and Materials Engineering (SCME), National University of Sciences and \\ Technology (NUST), Islamabad 44000, Pakistan \\ 4 Department of Materials, University of Oxford, 16 Parks Road, Oxford OX1 3PH, UK; \\ E-Mail: asad.hameed@materials.ox.ac.uk \\ 5 Department of Metallurgy and Materials Engineering, Pakistan Institute of Engineering and \\ Applied Sciences (PIEAS), Islamabad 45650, Pakistan; E-Mail: shaukat@pieas.edu.pk \\ * Author to whom correspondence should be addressed; E-Mails: mohammad.islam@gmail.com or \\ miqureshi@ksu.edu.sa; Tel.: +966-544-523-909; Fax: +966-114-670-199.
}

Received: 6 October 2013; in revised form: 4 November 2013 / Accepted: 7 November 2013 / Published: 5 December 2013

\begin{abstract}
Pure polyamide 6 (PA6) and polyamide 6/carbon nanotube (PA6/CNT) composite samples with 0.5 weight percent loading of pristine or functionalized CNTs were made using a solution mixing technique. Modification of nanotube surface as a result of chemical functionalization was confirmed through the presence of lattice defects as examined under high-resolution transmission electron microscope and absorption bands characteristic of carboxylic, sulfonic and amine chemical groups. Microstructural examination of the cryogenically fractured surfaces revealed qualitative information regarding CNT dispersion within PA6 matrix and interfacial strength. X-ray diffraction studies indicated formation of thermodynamically more stable $\alpha$-phase crystals. Thermogravimetric analysis revealed that CNT incorporation delayed onset of thermal degradation by as much as $70{ }^{\circ} \mathrm{C}$ in case of amine-functionalized CNTs, thus increasing thermal stability of the composites. Furthermore, addition of amine-functionalized CNTs caused an increase in crystallization and melting temperatures from the respective values of 177 and $213{ }^{\circ} \mathrm{C}$ (for neat PA6) to 211 and $230^{\circ} \mathrm{C}$ (for composite), respectively.
\end{abstract}


Keywords: polyamide 6; carbon nanotubes; nanocomposites; thermogravimetric analysis

\section{Introduction}

Due to their novel properties [1], both single- and multi-walled carbon nanotubes (CNTs) are considered potential candidates as filler materials for polymer composites with enhanced mechanical, electrical, thermal, and/or optical properties. The issues related to dispersion of CNTs in the polymer matrix and the formation of strong polymer/CNT interface for effective load transfer necessitate CNT surface modification. Defect functionalization, covalent functionalization, and non-covalent functionalization are among the main approaches to prevent CNT aggregation and to obtain good dispersion in the polymer matrix. While non-covalent functionalization routes do not induce any structural damage to the nanotube lattice, thus not adversely affecting physical properties, the efficiency of load transfer may be low due to weak forces between nanotube surface and the molecule wrapped around it. On the other hand, any oxidation treatment in air or acidic medium leads to attachment of $-\mathrm{COOH}$ or $-\mathrm{OH}$ functional groups, the removal of catalyst metal nanoparticles and the opening of nanotube ends.

Solution mixing is a relatively simple polymer composite processing technique that involves dispersion of the polymer and filler materials into a suitable solvent, dispersion through ultrasonic vibration followed by heating for solvent evaporation. This approach has been successfully employed for incorporating CNTs into polycarbonate, styrene-butadiene rubber, polyamide [2-4]. Polyamide 6 (PA6, polycarpolactam), a thermoplastic polymer with wide applications in construction and automobile industries, is a semi-crystalline polymer that offers advantages such as very good mechanical properties, excellent chemical stability, and low price compared to other polyamides. Carbon nanotubes have been reported to enhance percent crystalline character of PA6 by offering nucleation sites on the nanotubes walls $[5,6]$, the extent of which depends on nanotube type (single- or multi-walled), condition (as-received, purified, or functionalized), and content (loading in terms of weight percent) [7-9]. In addition to nanotubes, the crystallization kinetics and mechanical properties have been reported to be influenced by many factors such as processing technique employed, shear flow, temperature, mixing time, rotational speed, cooling rate, etc. [10,11].

Using a two-step grafting-from strategy, $\mathrm{Qu}$ et al. [12] made nylon6/single-walled nanotube samples through anionic ring-opening mechanism and characterized for their dispersion and thermal properties. Later, Yang et al. [13] adopted similar approach for multi walled carbon nanotubes (MWCNTs) using caprolactam functionalized CNTs and sodium caprolactamate as initiator and catalyst, respectively. By employing the use of sodium dodecylbenzene sulfonate as surfactant and very high shear rates to ensure uniform nanotubes dispersion, PA/CNT composites containing up to 3 wt \% CNTs were made and characterized for crystallinity and mechanical properties [14]. An improvement in degree of crystallinity through preferential nucleation of $\alpha$-phase crystals on nanotubes surface and enhanced mechanical properties in terms of increased storage modulus owing to the presence of an immobilized interface layer was reported in PA6/CNT composites prepared via the melt mixing technique [5]. Mixing and dispersion of carbon nanotubes is also reported to depend on 
the rotation speed, which in turn, influences induced shear stresses producing composites with higher storage modulus and complex viscosity [15].

In this paper, we present results from synthesis of neat PA6 and PA6/CNT composite samples through solution mixing technique. The effect of nanotubes incorporation, with and without surface modification, on dispersion, crystallization behavior and thermal stability of the resulting specimens was investigated. The dispersion characteristics were assessed from examination of cryogenically fractured surfaces. The effect of chemical modification was examined using high-resolution transmission electron microscope (HRTEM) and Fourier transform infra-red (FTIR) spectroscopy studies. The improvement in structure and thermal properties was assessed using X-ray diffraction (XRD) and differential scanning calorimetry (DSC) techniques.

\section{Experimental Section}

Multi walled carbon nanotubes (MWCNT) were purchased from Sun Nanotech Company limited (Nanchang, China) and used after purification (P-CNT) through $\mathrm{HCl}$ treatment or after a combination of oxidative/defects and covalent functionalization treatment to attach an amine (N-CNT) group. All the chemicals, such as hydrochloric acid $(\mathrm{HCl})$, sulfuric acid $\left(\mathrm{H}_{2} \mathrm{SO}_{4}\right)$, nitric acid $\left(\mathrm{HNO}_{3}\right)$, thionyl chloride $\left(\mathrm{SOCl}_{2}\right)$, tetrahydrofuran $\left(\mathrm{C}_{4} \mathrm{H}_{8} \mathrm{O}\right)$, ethylenediamine $\left[\mathrm{C}_{2} \mathrm{H}_{4}\left(\mathrm{NH}_{2}\right)_{2}\right]$ and formic acid $(\mathrm{HCOOH})$ were of analytical grade with $>99.9 \%$ purity were obtained from Sigma-Aldrich (Shanghai, China). Commercially available PA6 with brand name "Xiangyu" (Qingdao.Co., Qingdao, China) was procured and used without any further treatment.

Amine-functionalized CNTs (N-CNT) were produced through reflux treatment $\left(80{ }^{\circ} \mathrm{C}, 6 \mathrm{~h}\right)$ of MWCNT with $\mathrm{HCl}$ and subsequent addition into mixed solution $(1: 3 \mathrm{v} / \mathrm{v})$ of $\mathrm{HNO}_{3}$ and $\mathrm{H}_{2} \mathrm{SO}_{4}$ for ultrasonic vibration $(2 \mathrm{~h})$, magnetic stirring $\left(50{ }^{\circ} \mathrm{C}, 1 \mathrm{~h}\right)$ and reflux reaction $\left(85^{\circ} \mathrm{C}, 12 \mathrm{~h}\right)$. After washing the MWCNT/acid mixture, the filtrate was dried in oven $\left(110{ }^{\circ} \mathrm{C}, 12 \mathrm{~h}\right)$ and then heat treated $\left(350{ }^{\circ} \mathrm{C}, 0.5 \mathrm{~h}\right)$ for removal of acidic traces, if any. After grinding $(15 \mathrm{~min})$, the powder was added in $\mathrm{SOCl}_{2}$, sonicated $(1 \mathrm{~h})$ for dispersion and refluxed $\left(60^{\circ} \mathrm{C}, 24 \mathrm{~h}\right)$ for substitution of $-\mathrm{COOH}$ group by -OCl group. Following $\mathrm{SOCl}_{2}$ evaporation, excessive amount was removed using $\mathrm{C}_{4} \mathrm{H}_{8} \mathrm{O}$ followed by vacuum drying $\left(50{ }^{\circ} \mathrm{C}, 1 \mathrm{~h}\right)$. After mixing and dispersion of the dry powder in $\mathrm{C}_{2} \mathrm{H}_{4}\left(\mathrm{NH}_{2}\right)_{2}$, a reflux treatment was carried out $\left(85^{\circ} \mathrm{C}, 48 \mathrm{~h}\right)$ for attachment of amine group. N-CNTs were extracted through $\mathrm{C}_{2} \mathrm{H}_{4}\left(\mathrm{NH}_{2}\right)_{2}$ evaporation in vacuum, washing in ethanol and drying $\left(100{ }^{\circ} \mathrm{C}, 2 \mathrm{~h}\right)$.

To produce nanocomposite samples, the first step involved mixing $10 \mathrm{~g}$ of PA6 and certain amount of pristine CNT (P-CNT) in $50 \mathrm{~mL} \mathrm{HCOOH} \mathrm{(due} \mathrm{to} \mathrm{formic} \mathrm{acid} \mathrm{ability} \mathrm{to} \mathrm{dissolve} \mathrm{PA6} \mathrm{very} \mathrm{easily)} \mathrm{[16]}$ and magnetic stirring $\left(100{ }^{\circ} \mathrm{C}, 8 \mathrm{~h}\right)$. During mixing, the beaker was sealed with aluminum foil to ensure complete CNT dispersion into PA6 and to avoid solvent evaporation. After that, the foil was partially removed to evaporate the solvent until the mixture dried. Afterward, the dried residue was treated in a vacuum oven at $200{ }^{\circ} \mathrm{C}$ so that any traces of solvent were removed. In the same manner, composites with N-CNT were prepared. Finally, thin composite films were made from hot press at $235^{\circ} \mathrm{C}$ and 200 bar pressure. The PA6 samples denote neat polyamide 6 whereas composite samples are named as PA6/y-CNT where $y$ is $\mathrm{P}$ or $\mathrm{N}$.

Neat and composite PA6 samples were cryogenically fractured in liquid nitrogen and analyzed under scanning electron microscope (JEOL JSM 6460, Tokyo, Japan). Structural and phase analysis 
was carried out using X-ray diffraction (STOE Stadi MP, Darmstadt, Germany), while attachment of CNTs with PA6 matrix was confirmed using attenuation-total-reflection, Attenuated total reflectance Fourier transform infra-red spectroscope (JASCO ATR-FTIR-4100, Tokyo, Japan). Thermal analysis of the composites was done by operating thermogravimetric analyzer (TGA) (TA Instruments Pyris 1 diamond Q5000IR, New Castle, WA, USA) at rate of $10{ }^{\circ} \mathrm{C} / \mathrm{min}$. Thermal properties including melting point and crystallization temperature were measured under nitrogen flow using a differential scanning calorimeter (DSC) instrument (Mettler Toledo DSC 823e, Columbus, OH, USA).

\section{Results and Discussion}

\subsection{Microstructure Examination of Nanotubes and Composites}

The effect of acid treatment on CNT was assessed through HRTEM studies. The sidewall structures of pristine and chemically functionalized individual nanotubes are presented in Figure 1. The nanotubes have an outer diameter of $\sim 30 \mathrm{~nm}$ and are made up of several shells, as indicated by diffraction lines produced from coaxial shell configuration. The attachment of a functional group on the nanotube sidewalls is evident from discontinuity in the shell structure [17]. While HCl-treatment leads to the removal of metal catalyst nanoparticles (Figure 1b), further treatment for amine-functionalization causes formation of crystallographic defects beside covalent attachment of amine groups with acidic sites having hydroxyl, carbonyl, and carboxylic groups. The disruption of outer and near-surface shell structure, as manifested in Figure 1c, is indicative of strong interaction between chemical reagents and the MWCNT surface.

Figure 1. High-resolution transmission electron microscope (HRTEM) microstructures of the multi walled carbon nanotubes (MWCNTs): (a) Pristine CNT (P-CNT); (b) Carboxylic-functionalized CNT (C-CNT); and (c) Amine-functionalized CNT (N-CNT).
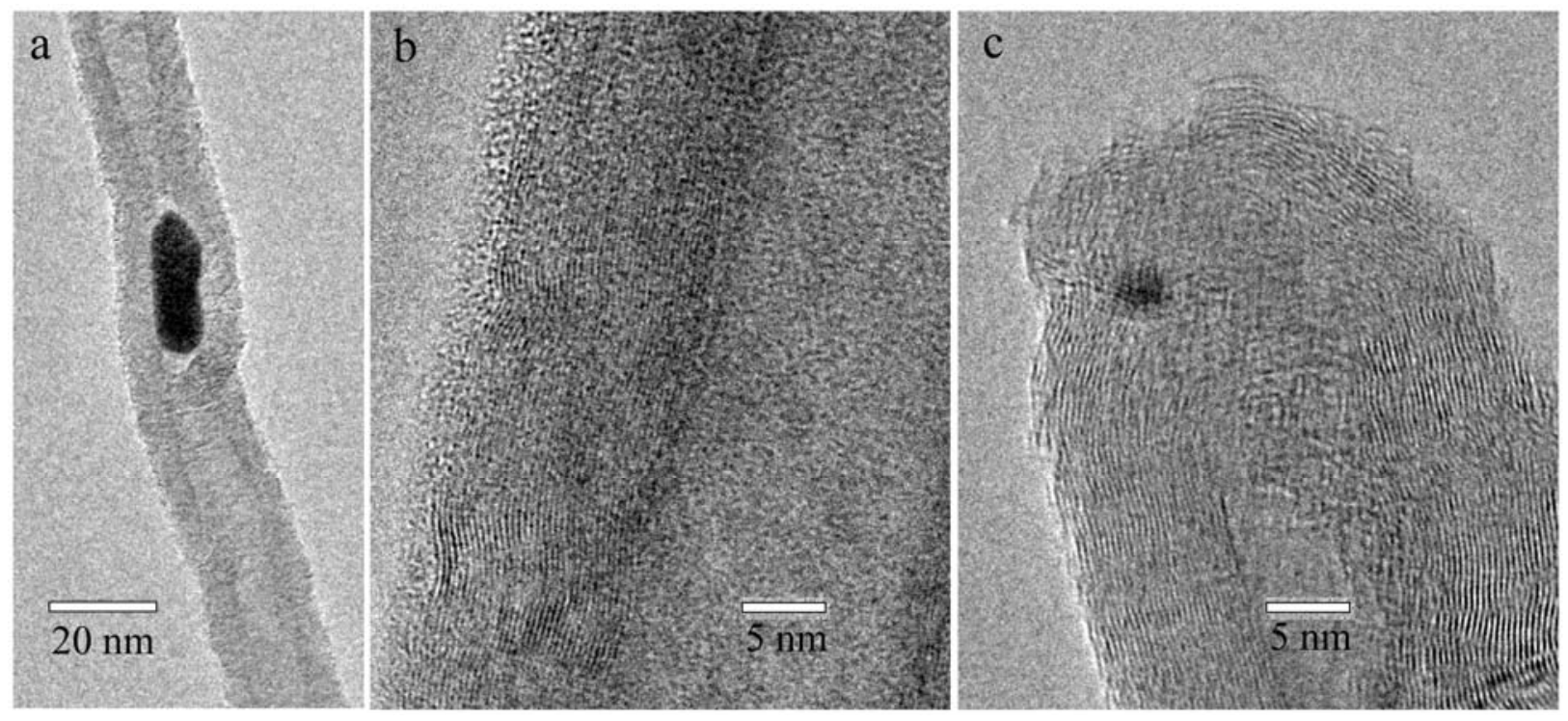

After neat and composite PA6 samples were cryogenically fractured in liquid nitrogen, microstructural examination was performed under scanning electron microscope (SEM) to investigate surface morphology and the effect of CNT incorporation. Low and high magnification images of 
the neat and composite (reinforced with pristine or amine-functionalized CNTs) PA6 surfaces after fracture are presented in Figure 2. SEM examination at low magnifications revealed that whereas neat PA6 underwent brittle fracture, nanocomposite samples had rough, grainy surface indicative of extensive deformation prior to failure. At high magnification (Figure 2c), distribution of P-CNT in PA6 matrix is clearly evident from filament-like features that appear to be protruding out of the polymer matrix. In case of PA6/0.5P-CNT, the width and length of these filaments are in the range of $\sim 110$ to $193 \mathrm{~nm}$ and few hundreds of nanometers, respectively. Similar features were observed in case of PA6/0.5N-CNT samples with width varying from 52 to $140 \mathrm{~nm}$. For same loading level, the extent of chemical functionalization dictates dispersion as well as interfacial strength with the PA6 matrix. Qualitatively, better CNT distribution was inferred from overall appearance of the composites that were of grey-black color in case of PA6/N-CNT composition, whereas PA6/P-CNT samples appeared matte black with inhomogeneous color. Functionalized CNTs usually exhibit better dispersion characteristics than P-CNT in both formic acid solution and the resulting nanocomposites [18,19]. A more comprehensive and quantitative information regarding effect of MWCNT incorporation can be derived from mechanical testing and dynamic thermal mechanical analysis of such nanocomposites samples.

Figure 2. SEM microstrucres of cryogenically fractured surfaces of neat and nanocomposite polyamide 6 (PA6) samples: (a,b) neat PA6; (c) PA6/0.5P-CNT; and (d) PA6/0.5N-CNT.

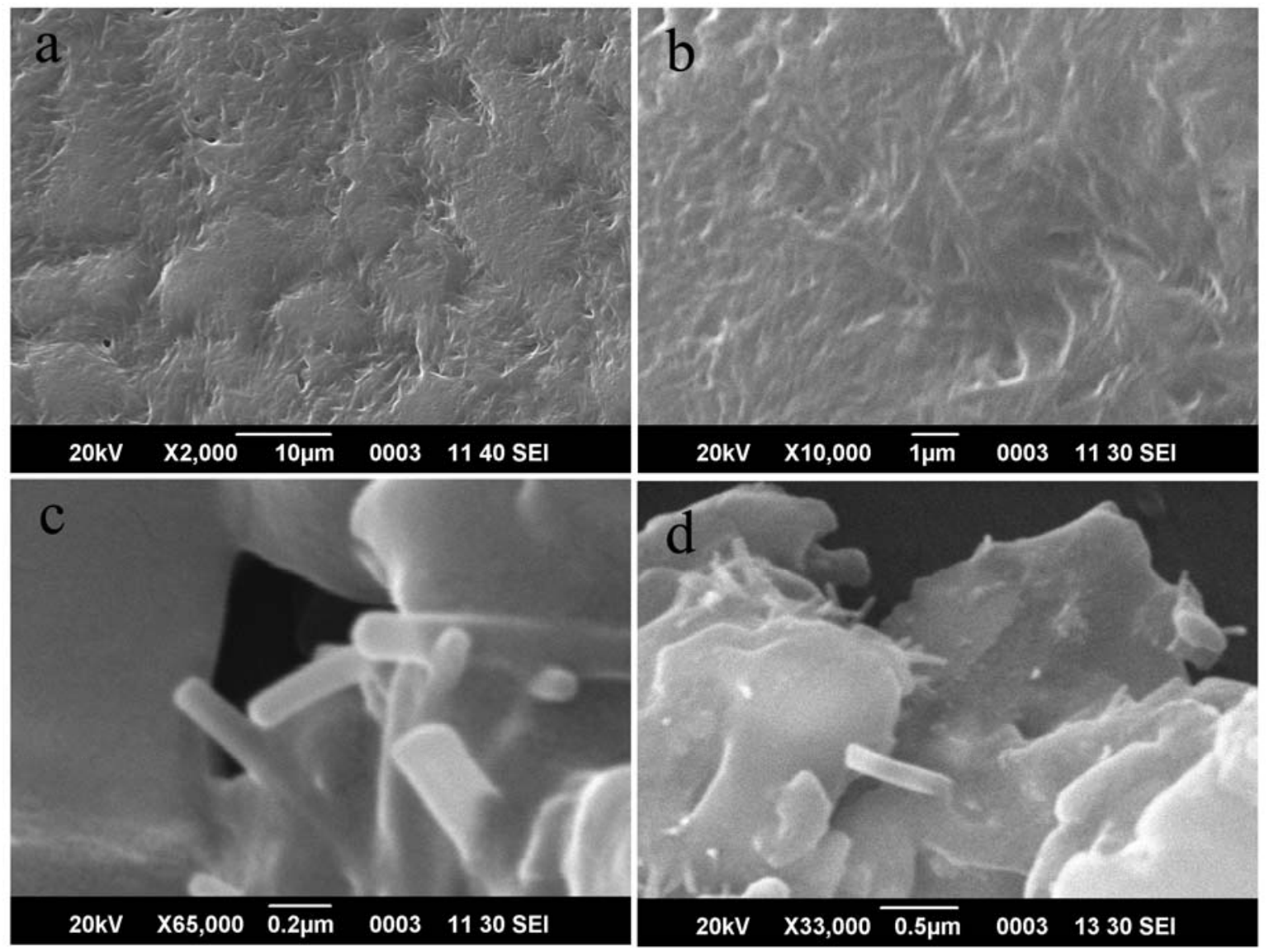




\subsection{Structural Studies}

X-ray diffraction spectra of the neat PA6 and nanocomposite samples are shown in Figure 3. As shown in Figure 3, while melt extrusion process produces PA6 with both $\alpha$ - and $\gamma$-form crystals present, the solution mixing route leads to $\alpha$-phase formation only, as indicated by the two peaks located at $2 \theta$ values of $19.8^{\circ}$ and $23.7^{\circ}$ with corresponding d-spacing of 4.43 and $3.7 \AA$ for (200) and [(002), (220)] planes, respectively. It is noteworthy that $\alpha$-phase is the thermodynamically stable phase consisting of sheets of hydrogen-bonded chains that are packed in an anti-parallel fashion whereas the $\gamma$-phase is the least stable phase formed due to random hydrogen bonding between parallel chains [7]. Little difference is observed between XRD patterns for neat and PA6/P-CNT composite sample. In the case of N-CNT incorporation, however, the enhanced degree of crystallanity is manifested by more intense peaks. Due to low CNT content, however, peaks characteristic of CNT do not appear on the XRD patterns. Thus, both the solution mixing processing route with formic acid as the solvent and the presence of pristine or functionalized CNT as potential sites for heterogeneous nucleation promote preferential growth of $\alpha$-form crystals in PA6/CNT composites. The crystallite size $(t)$ was determined using the Scherrer equation and was found to be $\sim 8.4 \mathrm{~nm}$ for the neat PA6 sample. While incorporation of N-CNT caused an increase in $t$ to $10.5 \mathrm{~nm}$, addition of P-CNT by the same amount led to a small drop in the $t$ value to $\sim 8 \mathrm{~nm}$. Thus, CNT incorporation into PA6 improves crystalline quality by offering more nucleation sites for crystallization.

Figure 3. X-ray diffraction patterns of neat PA6 (both from solution mixing and melt extrusion) and PA6/CNT nanocomposites.

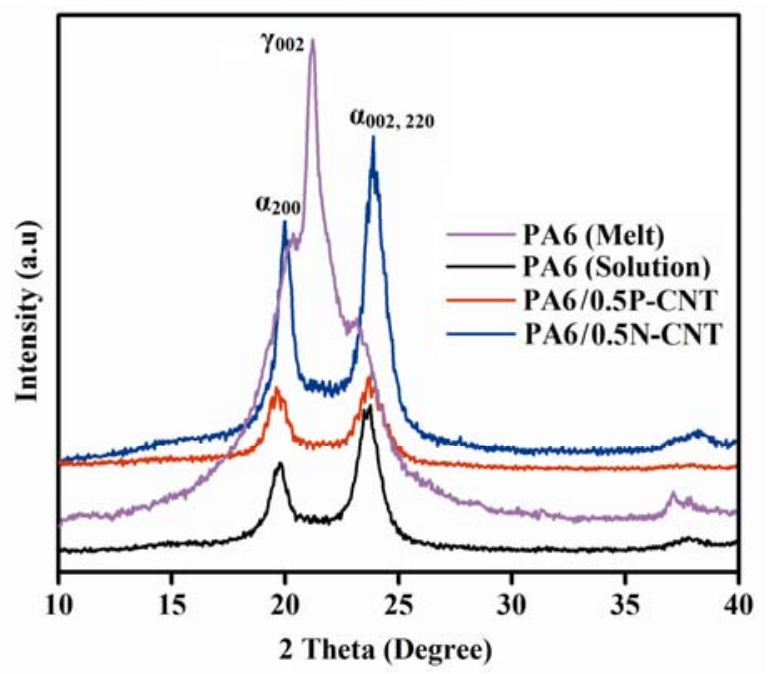

\subsection{Chemical Analysis}

Using the attenuated total reflectance Fourier transform infra-red spectroscope (ATR-FTIR), the spectra for neat PA6 as well as PA6 composites containing $0.5 \mathrm{wt} \%$ of amine-functionalized nanotubes were obtained, as shown in Figure 4. For the neat PA6 sample, absorption bands positioned at 1625,1530 and $1364 \mathrm{~cm}^{-1}$ can be assigned to the amide I, amide II and/or $\mathrm{H}-\mathrm{C}-\mathrm{H}$ asymmetric deformation and amide III band and/or H-C-H waging, respectively. Despite very small CNT content in composite samples, additional absorption bands characteristics of carboxylic, acyl and amine groups 
were noticed in FTIR spectra. The location of all the bands and their respective chemical bond assignments are given in Table 1. There was not much difference between spectra obtained for PA6 composites containing pristine or amine-functionalized nanotubes. Also, the presence of a weak absorption band at $1203 \mathrm{~cm}^{-1}$ confirmed formation of $\alpha$-form crystals [18], which corroborates the finding of the XRD studies.

Figure 4. FTIR spectra of Neat and PA6/CNT composite samples.

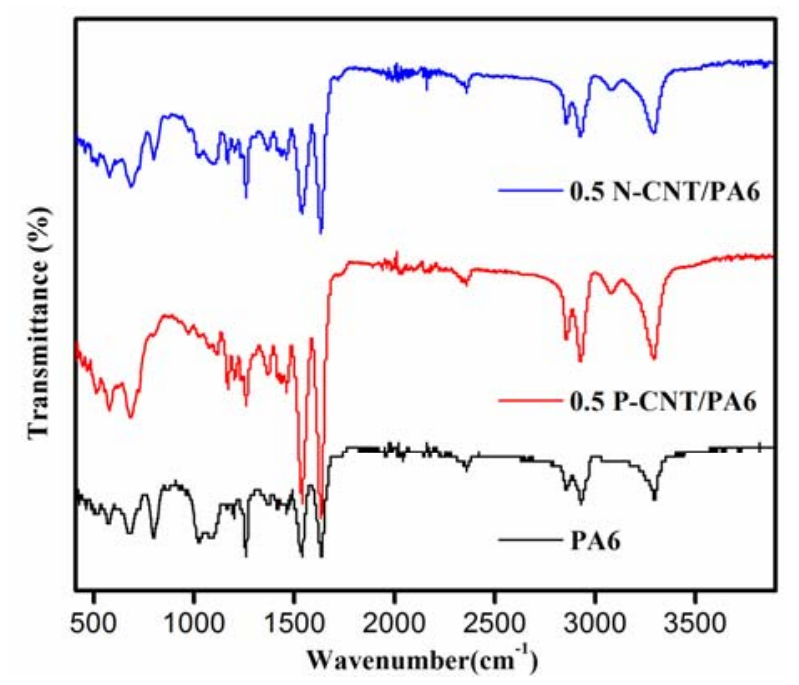

Table 1. Absorption bands and the chemical moieties responsible for their existence in neat PA6 and PA6/CNT composite samples [18,20,21].

\begin{tabular}{|c|c|c|}
\hline \multicolumn{2}{|c|}{ Band Position, $\mathrm{cm}^{-1}$} & \multirow{2}{*}{ Chemical group } \\
\hline Neat PA6 & PA6/CNT & \\
\hline $3290 \mathrm{~s}$ & 3290 & $\mathrm{~N}-\mathrm{H}$ stretching mode vibrations \\
\hline- & $3060 \mathrm{~m}$ & $\mathrm{C}-\mathrm{H}$ group asymmetric stretching \\
\hline $2931 \mathrm{~m}$ & 2931 & $\mathrm{H}-\mathrm{C}-\mathrm{H}$ group asymmetric stretching mode vibrations \\
\hline $2860 \mathrm{w}$ & 2860 & $\mathrm{H}-\mathrm{C}-\mathrm{H}$ group symmetric stretching mode vibrations \\
\hline $1635 \mathrm{vs}$ & 1635 & amide $\mathrm{I}(\mathrm{C}=\mathrm{O}$ stretching of quinone groups $)$ \\
\hline $1540 \mathrm{vs}$ & 1540 & $\begin{array}{c}\text { amide II (combination of } \mathrm{N}-\mathrm{H} \text { group bending and } \mathrm{C}-\mathrm{N} \text { group } \\
\text { stretching vibration) }\end{array}$ \\
\hline $1462 \mathrm{vw}$ & $1462 \mathrm{~s}$ & $\mathrm{C}=\mathrm{C}$ atomic stretching \\
\hline- & $1360 \mathrm{~m}$ & $\mathrm{C}-\mathrm{H}$ bending mode vibration \\
\hline $1260 \mathrm{vs}$ & 1260 & $-\mathrm{S}=\mathrm{O}$ sulfonic functional group, $\mathrm{C}-\mathrm{O}$ stretching frequency \\
\hline $1200 \mathrm{vw}$ & 1200 & $\mathrm{C}-\mathrm{C}-\mathrm{H}$ symmetric bending $/ \mathrm{CH}_{2}$ twisting \\
\hline- & $1170 \mathrm{w}$ & $\mathrm{C}=\mathrm{O}$ stretching vibration of carboxylic group \\
\hline $1070 \mathrm{~m}$ & 1070 & $\begin{array}{c}\mathrm{C}-\mathrm{O} \text { stretching vibrations (carboxylic group), }-\mathrm{OH} \text { groups on } \mathrm{CNT} \\
\text { surface after oxidation }\end{array}$ \\
\hline $1020 \mathrm{~m}$ & 1020 & $-\mathrm{SO}_{3} \mathrm{H}$ sulfonic groups \\
\hline 794 vs & - & $\mathrm{S}-\mathrm{O}$ group \\
\hline 712 & 730,712 & $\mathrm{~N}-\mathrm{H}$ wagging/ $\mathrm{CH}_{2}$ rocking \\
\hline $671 \mathrm{~s}$ & 666 & $\mathrm{C}-\mathrm{C}$ bending \\
\hline $570 \mathrm{w}$ & 570 & $\mathrm{O}=\mathrm{C}-\mathrm{N}$ bending \\
\hline
\end{tabular}

Notes: $\mathrm{s}=$ strong; $\mathrm{m}=$ medium; $\mathrm{v}=$ very $\mathrm{w}=$ weak. 


\subsection{Thermal Properties Measurement}

The effect of CNT incorporation on thermal stability of PA6 was assessed by heating neat and composite PA6 samples to $\sim 850{ }^{\circ} \mathrm{C}$ under air atmosphere. As shown in the thermographs presented in Figure 5, neat and composite PA6 exhibited similar behavior until $275^{\circ} \mathrm{C}$, after which thermal degradation occurred in a manner that was dependent upon sample composition. For neat PA6, samples prepared using solution processing route indicated a higher temperature of onset of decomposition than those produced via in situ polymerization [19] and melt blending process [7] which is characteristic of more stable $\alpha$-phase formed. Thus, our synthesis route involving extensive magnetic stirring at mildly high temperature of $100{ }^{\circ} \mathrm{C}$ produced more stable PA6 samples unlike those produced at higher temperatures, pressures, and/or sonication that may cause polymer chain fragmentation, formation of less stable $\gamma$-phase, or even reduction in CNT length.

Figure 5. Thermographs of neat and composite PA6 samples indicating weight loss as a function of temperature. The inset shows a magnified view of the overall thermograph.

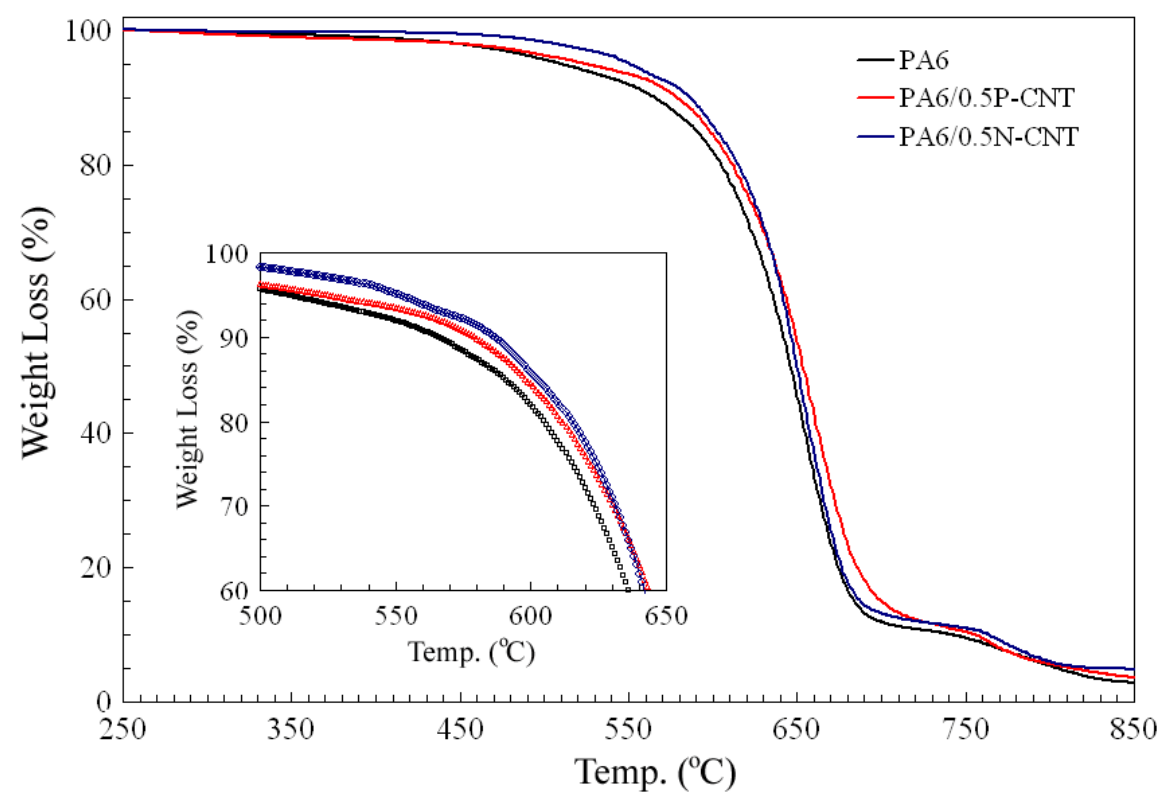

Comparison of neat and composite PA6 samples revealed improvement in thermal stability upon CNT incorporation of any type (pristine or functionalized) with maximum increase in onset of thermal degradation observed in case of PA6/N-CNT composition. The zoomed in view of the thermograph (Figure 5 inset), depicting thermal degradation behavior in the range of 430 to $630{ }^{\circ} \mathrm{C}$, clearly demonstrates that temperature indicative of initial decomposition increased by as much as $70{ }^{\circ} \mathrm{C}$. Whereas neat PA6 sample started to undergo thermal degradation at $\sim 450{ }^{\circ} \mathrm{C}$, PA6/N-CNT exhibited similar behavior once heated to $520^{\circ} \mathrm{C}$. Thermal decomposition resulted in $\sim 90$ percent loss of initial sample weight at temperatures in the range of $700-730{ }^{\circ} \mathrm{C}$, depending on sample composition. Another dip in the thermograph beyond a small plateau is anticipated to represent CNT decomposition.

Since thermo-oxidation is a well-known factor causing aging and degradation of PA6 in air, it is believed that CNT incorporation with homogeneous dispersion and strong PA6/CNT interfacial bonding reduced the extent of PA6 thermo-oxidation. 


\subsection{Melting and Crystallization Study}

The effect of P- or N-CNT addition on crystallization and melting behavior of PA6 was assessed using DSC. Thermograms showing the crystallization behavior of neat PA6 and PA6/CNT composites containing 0.5 weight percent of pristine- or amine-functionalized CNT are presented in Figure $6 \mathrm{a}$. While neat PA6 exhibits crystallization peak at $177.8^{\circ} \mathrm{C}$, an increase in crystallization temperature $\left(T_{\mathrm{C}}\right)$ by as much as $34{ }^{\circ} \mathrm{C}$ is observed in case of PA6/N-CNT sample. The $T_{\mathrm{C}}$ values for PA6/P-CNT and PA6/N-CNT composite samples were estimated to be 194.7 and $211.7^{\circ} \mathrm{C}$, respectively. The increase in $T_{\mathrm{C}}$ value upon MWCNT incorporation can be attributed to their role as a nucleating agent for the PA6 matrix. During melting of the PA6 and PA6/CNT nanocomposites, it is noticed that MWCNT incorporation increases the melting temperature $\left(T_{\mathrm{m}}\right)$ of the nanocomposite (Figure $6 \mathrm{~b}$ ). Furthermore, it is found that degree of increase in $T_{\mathrm{m}}$ is greater upon incorporation of amine functionalized MWCNT as compared to P-CNT. To varying degrees, the presence of MWCNT imposes restrictions on the motion of the PA6 chains upon heating, thus causing an increase in $T_{\mathrm{m}}$ by as much as $\sim 17{ }^{\circ} \mathrm{C}$ for PA6/0.5N-CNT nanocomposite.

Figure 6. (a) Crystallization thermograms and (b) melting thermograms for neat and composite PA6 samples at a rate of $10^{\circ} \mathrm{C} / \mathrm{min}$.
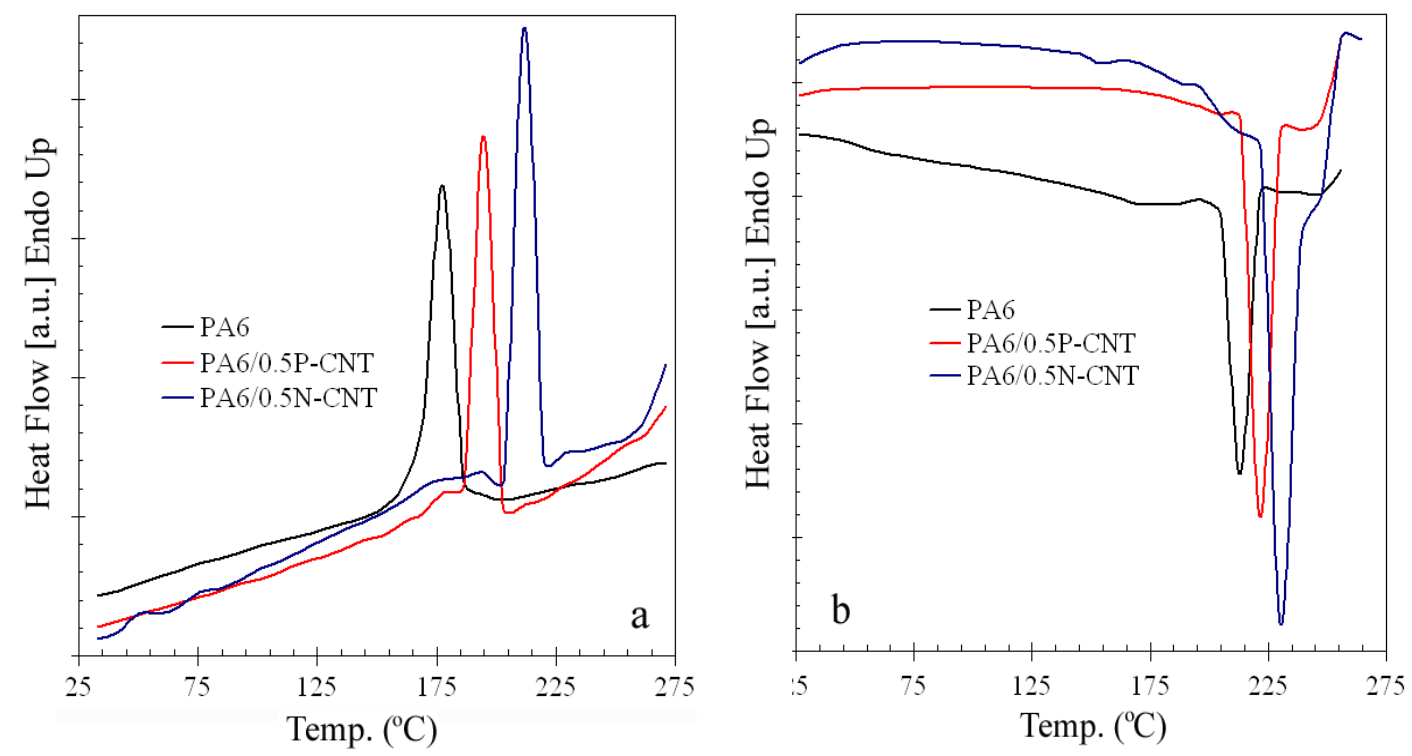

The results suggest that, in comparison with the melt extrusion process, solution mixing is a better technique since it promotes nucleation and growth of $\alpha$-form crystals (instead of less stable $\gamma$-phase) and causes little or no damage to polymer structure during processing, thus yielding higher $T_{\mathrm{C}}$ values. Secondly, carbon nanotubes, whether unmodified or modified through chemical functionalization, improve the crystalline nature of PA6 by offering nucleation sites on nanotubes sidewalls. In our study, the $T_{\mathrm{C}}$ value obtained from addition of $0.5 \mathrm{wt} \%$ amine-functionalized CNTs is in the same range as that reported for incorporation of $2 \mathrm{wt} \%$ pristine-CNTs using melt mixing [22] and higher than those reported for PA6 composites containing pristine or $\mathrm{COOH}$-functionalized nanotubes produced via the in situ polymerization process [23]. Also, functionalized nanotubes are better than pristine CNTs due to their better dispersion characteristics and strong PA6/CNT interfacial adhesion. 


\section{Conclusions}

Using the solution mixing technique, pure PA6 and PA6/CNT composites containing pristine or functionalized nanotubes with relatively low loading levels of $0.5 \mathrm{wt} \%$ were produced and characterized. The following conclusions were drawn from this work.

o The solution mixing process results in the formation of neat and composite PA6 samples containing $\alpha$-phase crystals only. The absence of thermodynamically less stable $\gamma$-phase implies better thermal, structural, and mechanical properties exhibited by the neat and composite samples.

o In all cases, addition of carbon nanotubes led to improvement in thermal properties. For a relatively low loading level of $0.5 \mathrm{wt} \%$, among the composites containing pristine or functionalized nanotubes, functionalized CNTs indicated superior properties.

The fact that the temperature indicative of onset of thermal degradation increased by $\sim 70{ }^{\circ} \mathrm{C}$ and $T_{\mathrm{C}}$ and $T_{\mathrm{m}}$ values increased by $\sim 44$ and $17{ }^{\circ} \mathrm{C}$ respectively, confirmed that PA6/N-CNT composite is the best among the different composite formulations explored due to chemical compatibility of amine-functionalized carbon nanotubes with PA6 with better dispersion and interfacial characteristics.

\section{Acknowledgments}

The authors would like to extend their sincere appreciation to the Deanship of Scientific Research at King Saud University (Riyadh, Saudi Arabia) for its funding of this research through the Research Group Project no. RGP-VPP-283. The authors also thank Nicolas Gautier from Institut des Matériaux Jean Rouxel, Nantes, France and Miss. Rabia Jamil at Institute of Chemistry, Quaid-e-Azam University (QAU), Islamabad, Pakistan for technical assistance with HRTEM and DSC studies.

\section{Conflicts of Interest}

The authors declare no conflict of interest.

\section{References}

1. Sahoo, N.G.; Rana, S.; Cho, J.W.; Chen, S.H.; Li, L. Polymer nanocomposites based on functionalized carbon nanotubes. Prog. Polym. Sci. 2010, 35, 837-867.

2. Li, C.; Pang, X.J.; Qu, M.Z.; Zhang, Q.T.; Wang, B.; Zhang, B.L.; Yu, Z.-L. Fabrication and characterization of polycarbonate/carbon nanotubes composites. Compos. A 2006, 37, 1485-1489.

3. Zhou, X.; Zhu, Y.; Liang, J.; Yu, S. New fabrication and mechanical properties of styrene-butadiene rubber/carbon nanotubes nanocomposite. J. Mater. Sci. Technol. 2010, 26, 1127-1132.

4. Dasari, A.; Yu, Z.Z.; Mai, Y.W. Electrically conductive and super-tough polyamide-based nanocomposites. Polymer 2009, 50, 4112-4121.

5. Logakis, E.; Pandis, C.; Peoglos, V.; Pissis, P.; Stergiou, C.; Pionteck, J.; Potschke, P.; Micusik, M.; Omastova, M. Structure-property relationships in polyamide $6 /$ multi-walled carbon nanotubes nanocomposites. J. Polym. Sci. B 2009, 47, 764-774. 
6. Bose, S.; Khare, R.A.; Moldenaers, P. Assessing the strengths and weaknesses of various types of pre-treatments of carbon nanotubes on the properties of polymer/carbon nanotubes composites: A critical review. Polymer 2010, 51, 975-993.

7. Liu, T.; Phang, I.Y.; Shen, L.; Chow, S.Y.; Zhang, W.D. Morphology and mechanical properties of multiwalled carbon nanotubes reinforced nylon-6 composites. Macromolecules 2004, 37, 7214-7222.

8. Caamaño, C.; Grady, B.; Resasco, D.E. Influence of nanotube characteristics on electrical and thermal properties of MWCNT/polyamide 6,6 composites prepared by melt mixing. Carbon 2012, 50, 3694-3707.

9. Mahmood, N.; Islam, M.; Hameed, A.; Saeed, S.; Khan, A.N. Polyamide-6-based composites reinforced with pristine or functionalized multi-walled carbon nanotubes produced using melt extrusion technique. J. Compos. Mater. 2013, doi:10.1177/0021998313484779.

10. Pegel, S.; Pötschke, P.; Petzold, G.; Alig, I.; Dudkin, S.M.; Lellinger, D. Dispersion, agglomeration, and network formation of multiwalled carbon nanotubes in polycarbonate melts. Polymer 2008, 49, 974-984.

11. Wang, X.; Bradford, P.D.; Liu, W.; Zhao, H.; Inoue, Y.; Maria, J.P.; Li, Q.; Yuan, F.G.; Zhu, Y. Mechanical and electrical property improvement in CNT/nylon composites through drawing and stretching. Compos. Sci. Technol. 2011, 71, 1677-1683.

12. Qu, L.; Veca, L.M.; Lin, Y.; Kitaygorodskiy, A.; Chen, B.; McCall, A.M.; Connell, J.W.; Sun, Y.-P. Soluble nylon-functionalized carbon nanotubes from anionic ring-opening polymerization from nanotube surface. Macromolecules 2005, 38, 10328-10331.

13. Yang, M.; Gao, Y.; Li, H.; Adronov, A. Functionalization of multiwalled carbon nanotubes with polyamide 6 by anionic ring-opening polymerization. Carbon 2007, 45, 2327-2333.

14. Song, R.; Yang, D.; He, L. Preparation of semi-aromatic polyamide (PA)/multi-wall carbon nanotube (MWCNT) composites and its dynamic mechanical properties. J. Mater. Sci. 2008, 43, 1205-1213.

15. Pötschke, P.; Villmow, T.; Krause, B. Melt mixed PCL/MWCNT composites prepared at different rotation speeds: Characterization of rheological, thermal, and electrical properties, molecular weight, MWCNT macrodispersion, and MWCNT length distribution. Polymer 2013, 54, 3071-3078.

16. Selling, G.W.; Biswas, A. Blends of zein and nylon 6. J. Polym. Environ. 2012, 20, 631-637.

17. Datsyuk, V.; Kalyva, M.; Papagelis, K.; Parthenios, J.; Tasis, D.; Siokou, A.; Kallitsis, I.; Galiotis, C. Chemical oxidation of multiwalled carbon nanotubes. Carbon 2008, 46, 833-840.

18. Deng, H.; Bilotti, E.; Zhang, R.; Wang, K.; Zhang, Q.; Peijs, T.; Fu, Q. Improving tensile strength and toughness of melt processed polyamide 6/multiwalled carbon nanotube composites by in situ polymerization and filler surface functionalization. J. Appl. Polym. Sci. 2011, 120, 133-140.

19. Vaisman, L.; Marom, G.; Wagner, H.D. Dispersions of surface-modified carbon nanotubes in water-soluble and water-insoluble polymers. Adv. Funct. Mater. 2006, 16, 357-363.

20. Ma, P.C.; Siddiqui, N.A.; Marom, G.; Kim, J.K. Dispersion and functionalization of carbon nanotubes for polymer-based nanocomposites: A review. Compos. A 2010, 41, 1345-1367.

21. Kakade, B.A.; Pillai, V.K. An efficient route towards the covalent functionalization of single walled carbon nanotubes. Appl. Surf. Sci. 2008, 254, 4936-4943. 
22. Saeed, K.; Park, S.Y. Preparation of multiwalled carbon nanotube/nylon- 6 nanocomposites by in situ polymerization. J. Appl. Polym. Sci. 2007, 106, 3729-3735.

23. Krause, B.; Pötschke, P.; Häußler, L. Influence of small scale melt mixing conditions on electrical resistivity of carbon nanotube-polyamide composites. Compos. Sci. Technol. 2009, 69, 1505-1515.

(C) 2013 by the authors; licensee MDPI, Basel, Switzerland. This article is an open access article distributed under the terms and conditions of the Creative Commons Attribution license (http://creativecommons.org/licenses/by/3.0/). 